Original paper

\title{
Collagen-driven remodeling of the intrahepatic duct wall in the PCK rat model of polycystic kidney disease-Caroli syndrome
}

\author{
Rithika Narayan ${ }^{1}$, Jingsong Li², Anthony J. Pellicano², Itzhak D. Goldberg² \\ 'Department of Science, Elwood John H. Glenn High School, New York, USA \\ ${ }^{2}$ Angion Biomedica Corp., New York, USA
}

\begin{abstract}
Aim of the study: Autosomal dominant polycystic kidney disease (ADPKD) is characterized by formation and expansion of cysts within the kidney. Caroli syndrome (CS) is characterized by cystic saccular dilatation of intrahepatic ducts. Kidney and liver images from a model of ADPKD-CS were evaluated to characterize remodeling of the cystically dilated intrahepatic duct wall and the renal cyst wall.

Material and methods: Archival digitized images from Masson's trichrome-stained renal and Picrosirius red (PSR)-stained renal and hepatic cross-sections were sourced from the PCK rat model of ADPKD-CS, and agematched Sprague-Dawley rats (wild-type). Cross-sectional areas and wall thicknesses of renal cysts and intrahepatic ducts were measured. Circularly polarized PSR microscopy was utilized to observe accumulation of collagen and identify its subtype.

Results: In the PCK rat model of ADPKD-CS, renal cysts were relatively thin-walled in comparison to intrahepatic ducts with renal cyst cross-sectional area to wall ratio 47-fold greater than the corresponding ratio in intrahepatic ducts. Increasing intrahepatic duct cross-sectional area was accompanied by a rapid and steep rise in wall thickness. There was a weak but significant direct correlation $(r=0.49, p=0.037)$ between renal cyst cross-sectional area and wall thickness. Circularly polarized Picrosirius red microscopy revealed collagen I accumulation within the walls of dilated intrahepatic ducts but not renal cysts.
\end{abstract}

Conclusions: These data suggest that unlike renal cysts, cystically dilated intrahepatic ducts undergo collagen-driven wall remodeling in the PCK rat.

Key words: Caroli syndrome, polycystic kidney, cyst, duct, collagen.

\section{Address for correspondence}

Rithika Narayan, Department of Science, Elwood John H. Glenn High School, 478 Elwood Rd, East Northport, NY 11731, USA, e-mail: narayanr@elwood.k12.ny.us

\section{Introduction}

Autosomal dominant polycystic kidney disease (ADPKD) is caused by mutations in the polycystic kidney disease 1 (PKD1) or 2 (PKD2) gene and is characterized by formation and enlargement of fluid-filled cysts in the kidneys [1]. Caroli syndrome (CS), also a rare disease, is genetically acquired via a mutation in chromosome 6 (polycystic kidney and hepatic disease 1 (PKHD1) gene), and is characterized by cystic saccular dilatation of fluid-filled intrahepatic ducts [2].
Renal cysts have been reported to rupture, causing a host of complications including hematuria, peritonitis, septic shock and even death [3-6]. By contrast, rupture of cystically dilated intrahepatic ducts has not been reported.

The PCK rat carries a mutation in the PKHD1 gene but phenotypically resembles ADPKD and CS $[7,8]$. Kidney and liver images from PCK rats were evaluated to test the hypothesis that the cystically dilated intrahepatic duct wall but not the renal cyst wall undergoes adaptive remodeling. 


\section{Material and methods}

\section{Image analysis}

Archival digitized images from Masson's trichrome-stained renal and Picrosirius red (PSR)-stained renal and hepatic cross-sections $(0.5 \times, 4 \times$ or $10 \times)$ were sourced from a drug-naive male PCK rat (7 months) and age-matched male Sprague-Dawley rats (wildtype) from a previous study [8]. Renal cysts $(n=18)$ were examined from 9 PCK rats; wild-type kidneys were cyst-free. Intrahepatic ducts $(n=26)$ from 9 wild-type animals were examined; $n=28$ intrahepatic ducts were examined from 8 PCK rats. Images were analyzed using ImageJ. Wall thickness was measured at 3 randomly selected but distinct loci along the cyst and duct walls, averaged, and expressed in $\mathrm{mm}$; renal cyst cross-sectional area and intrahepatic duct cross-sectional area were expressed in $\mathrm{mm}^{2}$. Circularly polarized PSR microscopy was utilized to observe accumulation of collagen and identify its subtype based on the birefringence pattern [9].

\section{Statistical analysis}

Data are expressed as average \pm standard error of mean. Between-group differences were calculated using Student's $t$-test and a $p$-value $<0.05$ was assumed significant. Correlations between cross-sectional area of the cyst or duct and its wall thickness were made and curve-fit using Microsoft Excel. Renal cyst and intrahepatic duct collagen content was expressed as a percentage of the field area under observation.

\section{Results}

Livers from the wild-type cohort exhibited normal intrahepatic ducts (Fig. 1A, B) whereas livers from PCK rats exhibited cystically dilated intrahepatic ducts (Fig. 1A, C). Cross-sectional area of the intrahepatic duct was $\sim 5$-fold greater in the PCK cohort vs. the wild-type cohort (Fig. 1D). Nevertheless, duct wall thickness was more pronounced, $\sim 27$-fold greater, in the PCK liver compared to the wild-type cohort (Fig. 1E). Thus, the ratio of duct cross-sectional area to wall thickness was reduced in the PCK liver compared to the wild-type liver (Fig. 1F).

The renal parenchyma of wild-type animals was cyst-free (Fig. 2A, B), whereas PCK rat kidneys were punctuated with numerous cysts enclosed by relatively thin walls (Fig. 2A, C). In fact, the cyst wall was ruptured in one or more places in many of the larger renal cysts (Fig. 2D, E, arrows). Cross-sectional areas and cross-sectional area to wall ratio of intrahepatic ducts (wild-type and PCK) and renal cysts (PCK) were compared. Renal cysts had much larger cross-sectional areas than intrahepatic ducts (Fig. 3A). Equally striking, cross-sectional area to wall ratio of renal cysts was multiple times larger than that of the ducts (Fig. 3B).

To determine the relation between cross-sectional area of a duct or cyst and its wall thickness, these variables were plotted against each other. Intrahepatic duct wall thickness rose rapidly as a function of duct cross-sectional area and subsequently plateaued, a relation best described by a logarithmic function (Fig. 4A). In renal cysts, increasing cross-sectional area was accompanied by a slowly progressing (slope $=0.0028$ ) increase in wall thickness (Fig. 4B). The linear correlation between these variables in renal cysts was weak but significant $(r=0.49, p=0.037)$.

Circular polarized PSR microscopy was used to determine wall composition of intrahepatic ducts and renal cysts. Duct walls appeared a bright yellow-orange (Fig. 5A, B) characteristic of collagen I deposition (Fig. 5E). By contrast, no collagen deposition was evident along renal cyst walls (Fig. 5C-E).

\section{Discussion}

The PCK rat model of ADPKD-CS is characterized by the presence of multiple renal cysts and cystically dilated intrahepatic ducts. Compared to the cyst wall, the duct wall is relatively thick. Intrahepatic duct wall thickness increases rapidly as a function of increasing cross-sectional area and is driven by accumulation of collagen I. By contrast, renal cyst wall thickness exhibits a weak correlation with cross-sectional area with no collagen accumulation.

Adult ADPKD patients are at risk of hemorrhage, peritonitis, sepsis and even death from rupture of enlarged fluid-filled renal cysts. By contrast, there are no reports of rupture of cystically dilated intrahepatic ducts in CS. The present study conducted in the PCK rat model of ADPKD-CS was designed to test the hypothesis that cystically dilated intrahepatic ducts but not renal cysts undergo adaptive wall remodeling. Compared to age- and gender-matched wild-type rat livers, intrahepatic ducts in the PCK cohort had much larger cross-sectional areas. Nevertheless, the increase in duct cross-sectional area was accompanied by a rapid increase in duct wall thickness. As a result, the cross-sectional area to wall thickness ratio was smaller in PKC rat ducts compared to the wild-type cohort. Interestingly, this rapid increase in duct wall thickness plateaued, suggesting that adequate structural support had been achieved. While wild-type kidneys had no 

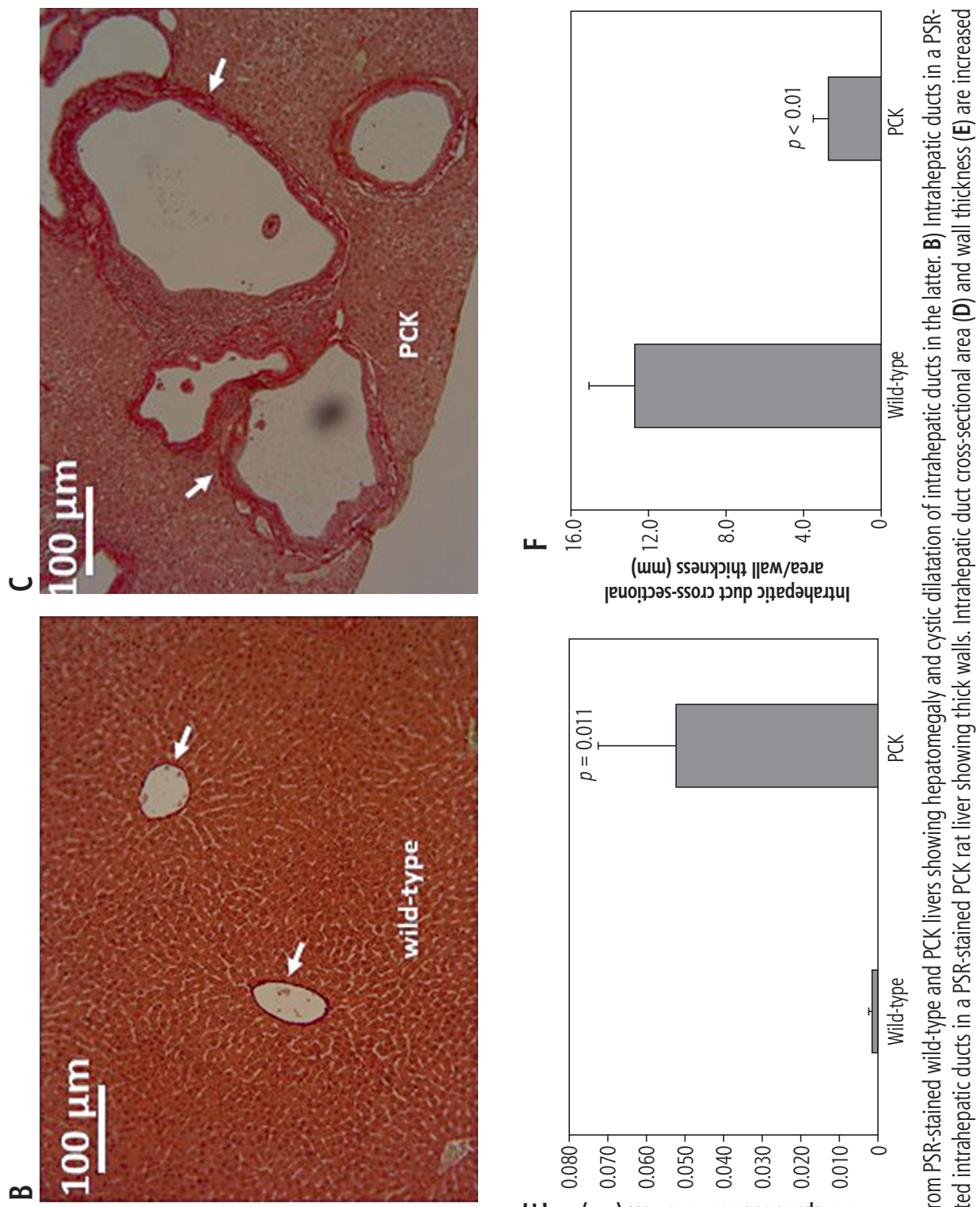

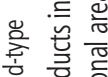

突

응

.를

능

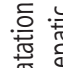

蔩 $\frac{8}{\frac{0}{0}}$

㫐

范

들

斊尊

뭉

产

중은

ㄴ.

总总

范 잉

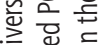

弟焉 兽

은 홍 행

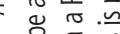

空.气

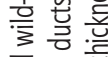

믈 弟

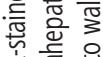

范苛

咅

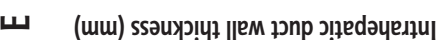
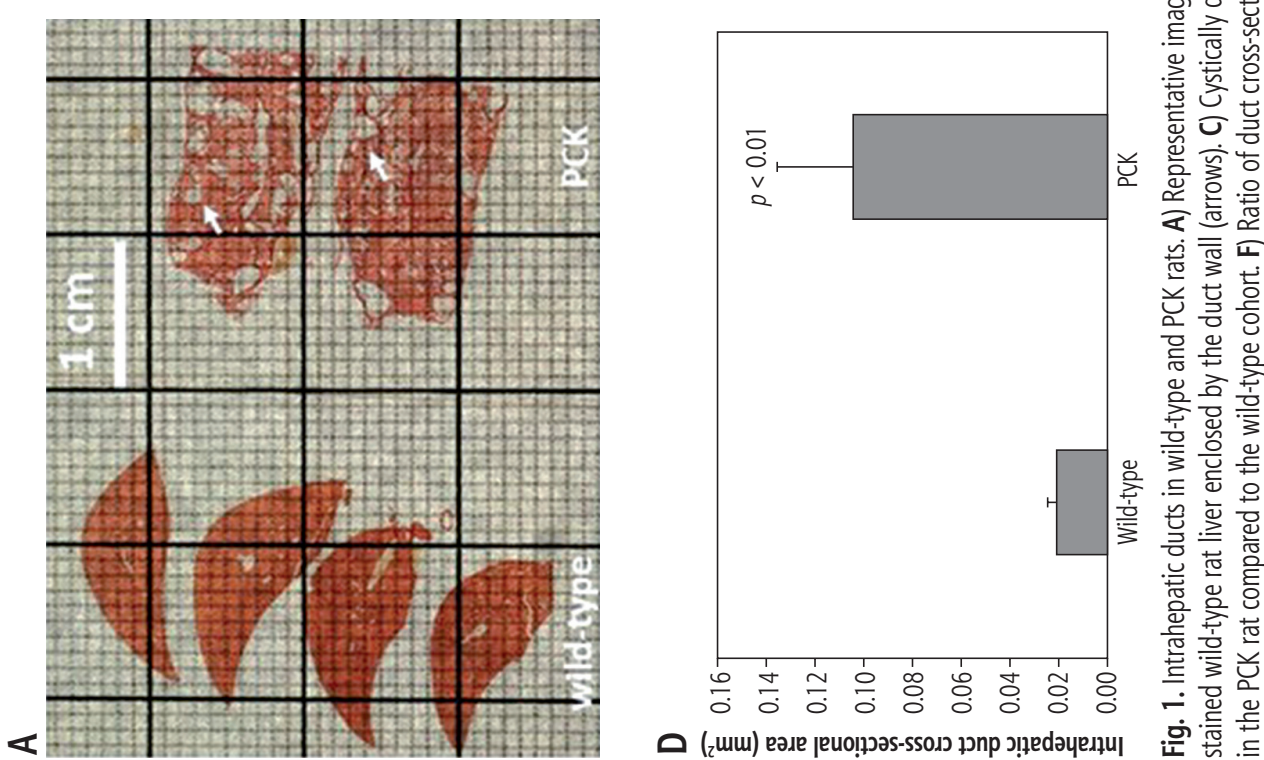

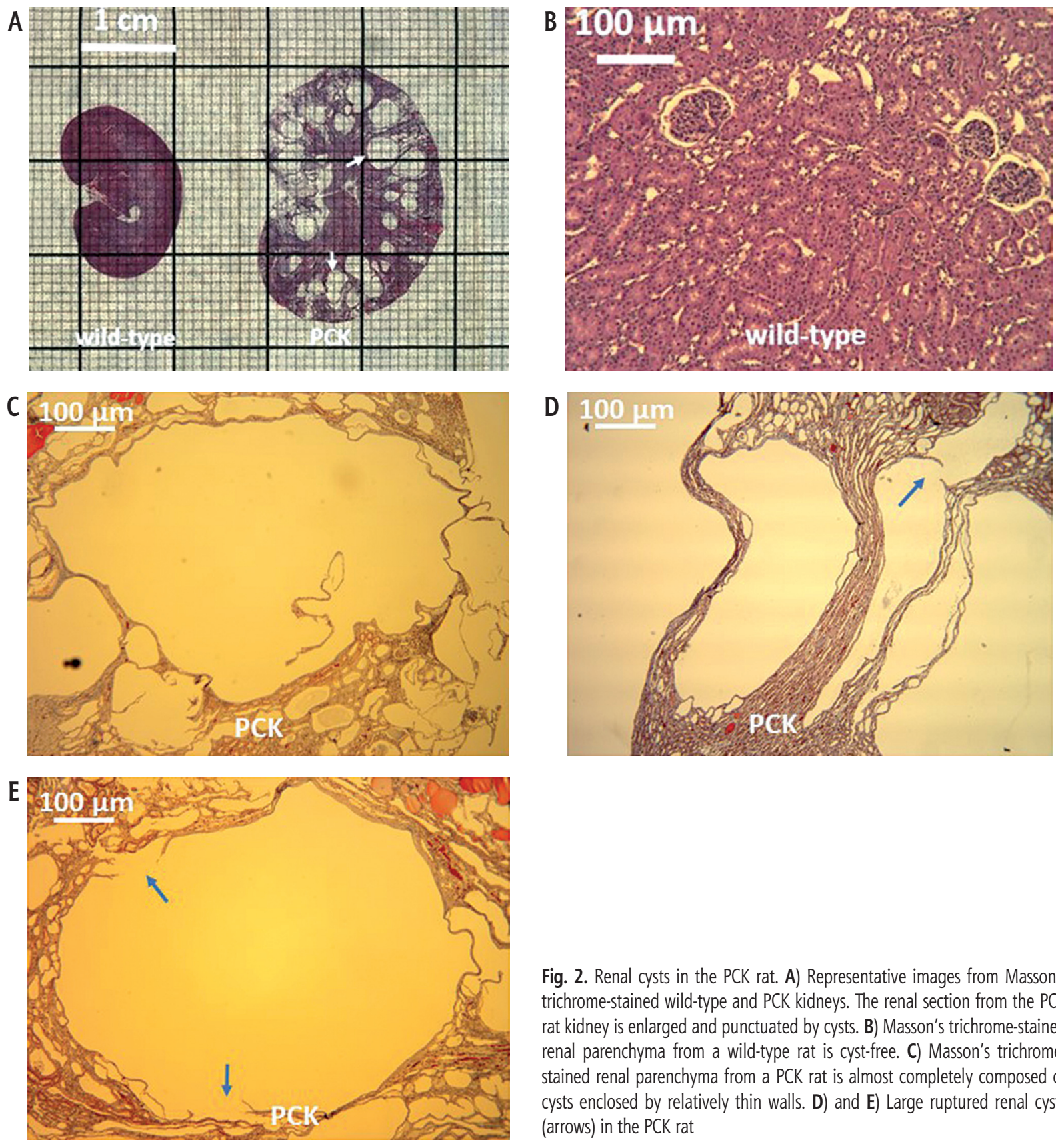

Fig. 2. Renal cysts in the PCK rat. A) Representative images from Masson's trichrome-stained wild-type and PCK kidneys. The renal section from the PCK rat kidney is enlarged and punctuated by cysts. B) Masson's trichrome-stained renal parenchyma from a wild-type rat is cyst-free. C) Masson's trichromestained renal parenchyma from a PCK rat is almost completely composed of cysts enclosed by relatively thin walls. D) and $\mathbf{E}$ ) Large ruptured renal cysts (arrows) in the PCK rat
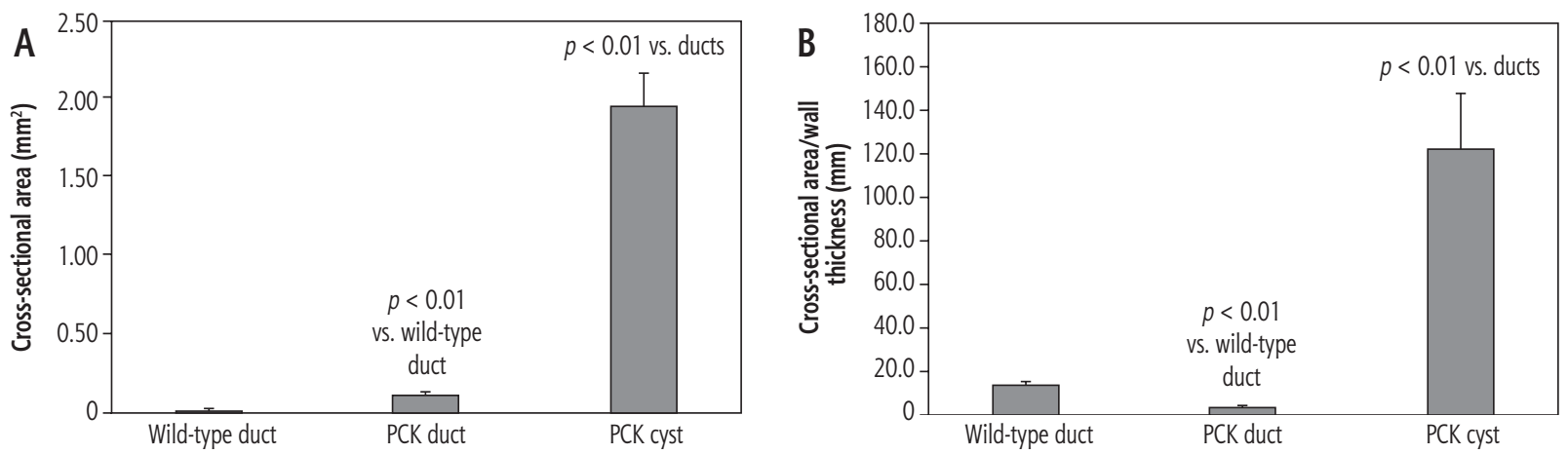

Fig. 3. Duct and cyst characteristics. A) Cross-sectional area of the renal cyst in the PCK rat is multiples times greater than that of the ducts in wild-type and PCK livers. B) Ratio of cross-sectional area to wall thickness is largest in the PCK renal cyst 

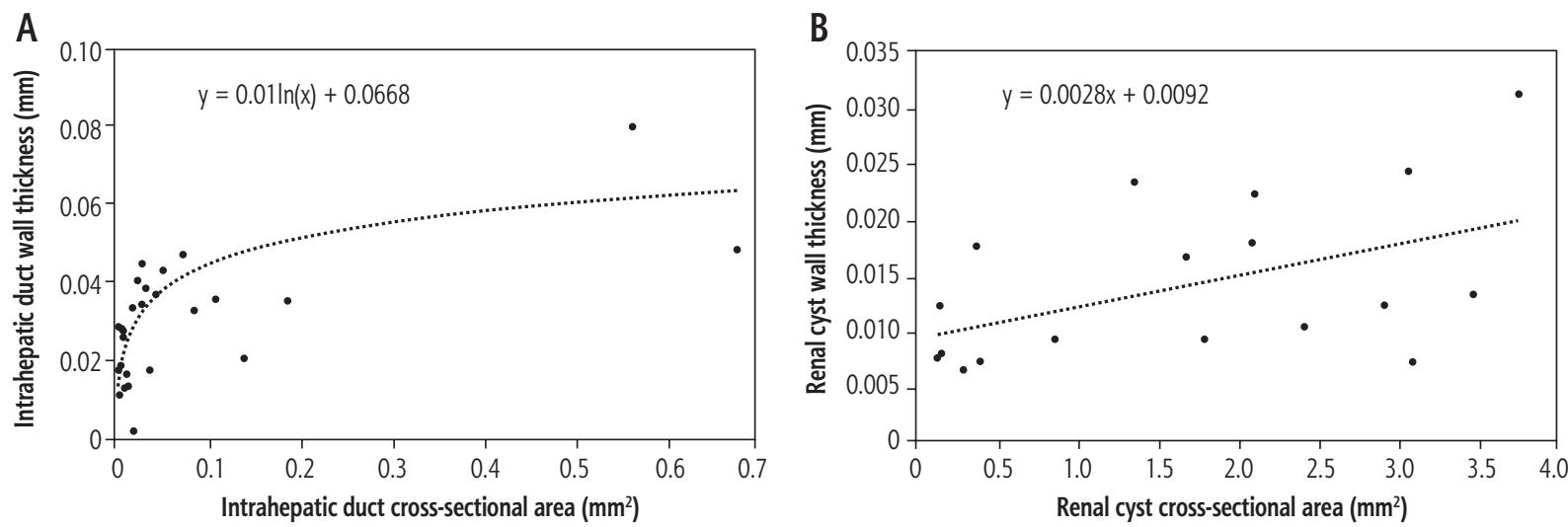

Fig. 4. Duct and cyst wall remodeling. A) As intrahepatic duct cross-sectional area increases, duct wall thickness increases rapidly before plateauing. B) There is a weak but significant direct correlation between renal cyst cross-sectional area and wall thickness
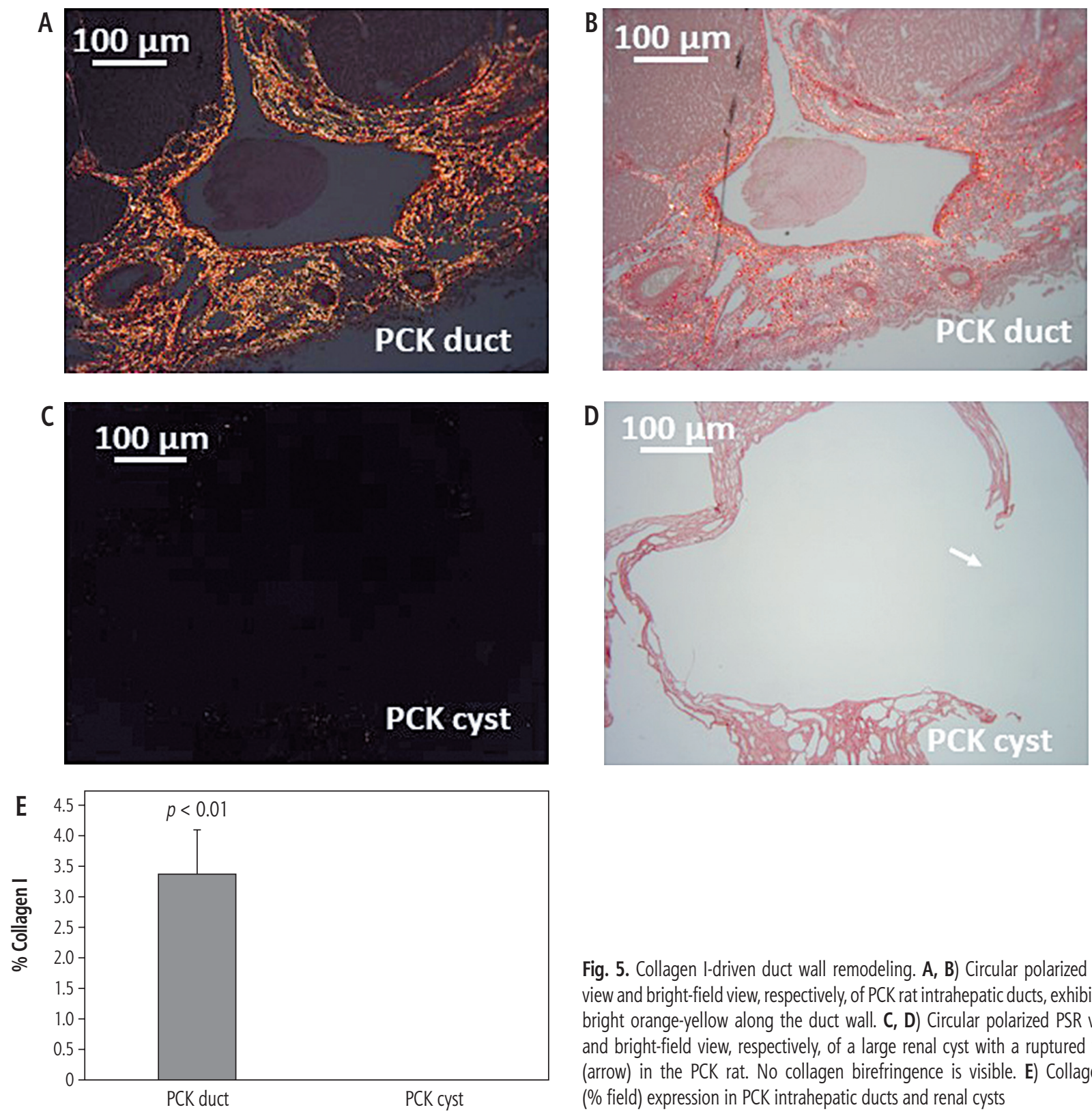

Fig. 5. Collagen I-driven duct wall remodeling. A, B) Circular polarized PSR view and bright-field view, respectively, of PCK rat intrahepatic ducts, exhibiting bright orange-yellow along the duct wall. C, D) Circular polarized PSR view and bright-field view, respectively, of a large renal cyst with a ruptured wall (arrow) in the PCK rat. No collagen birefringence is visible. E) Collagen I (\% field) expression in PCK intrahepatic ducts and renal cysts 
cysts, the PKC rat exhibited multiple small and large cysts consistent with the ADPKD phenotype. Renal cysts tended to be larger than the intrahepatic ducts in the PCK rat. Nevertheless, relative to cross-sectional area, cyst walls were significantly thinner than their ductal counterparts. Furthermore, as cyst cross-sectional area increased, wall thickness increased only gradually, evidenced by the shallow slope of this relationship. Interestingly, many of the larger cysts were ruptured in one or more places, whereas none of the intrahepatic ducts were ruptured. Consistent with, and as a consequence of Laplace's law of wall tension [10], it is possible that the renal cyst wall cannot tolerate the tension experienced by it, causing it to rupture.

Collagen is a major structural component of cellular organelles. The PSR stain is one of the best understood histochemical techniques able to selectively highlight collagen networks. Relatively inexpensive, the technique relies on the birefringent properties of collagen molecules. While the PSR stain alone does not selectively bind the collagen network, it becomes more specific than the other common collagen stains when combined with polarized light detection $[9,11]$. Under circularly polarized PSR microscopy, thin bundles of collagen type III appear green-yellow, whereas large bundles of tightly packed, well-organized and mature collagen type I molecules appear yellow-orange [9]. Use of circularly polarized PSR microscopy revealed bundles of yellow-orange collagen I $[9,11]$ along the intrahepatic duct walls, indicating the presence of mature collagen type I, which provides both structural support and tensile strength [9]. By contrast, there was no accumulation of collagen I or collagen III along the renal cyst walls. Together these data suggest that collagen I-driven remodeling of their walls stabilizes cystically dilated intrahepatic ducts, a mechanism that appears absent in renal cysts.

In as much as the PCK rat is a model of ADPKD$\mathrm{CS}$, these findings suggest that renal cysts are at increased risk for rupture, whereas cystically intrahepatic ducts do not, as the latter undergo remodeling to accommodate increased wall tension. Needless to say, this finding remains to be confirmed in human subjects. Nevertheless, these data suggest that ADPKD patients at risk for rupture of renal cysts may benefit from therapies including cystocentesis or aspiration of fluid from the cysts, especially the larger cysts, and/or sclerotherapy $[12,13]$ to relieve wall tension.

\section{Disclosure}

The authors report no conflict of interest.

\section{References}

1. Bergmann C, Guay-Woodford LM, Harris PC, et al. Polycystic kidney disease. Nat Rev Dis Primers 2018; 4: 50.

2. Kim JT, Hur YH, Park JM, et al. Caroli's syndrome with autosomal recessive polycystic kidney disease in a two month old infant. Yonsei Med J 2006; 47: 131-134.

3. Nishiura JL, Eloi SR, Heilberg IP. Pain determinants of pain in autosomal dominant polycystic kidney disease. J Bras Nefrol 2013; 35: 242-243.

4. Xue C, Zhou CC, Wu M, et al. The clinical manifestation and management of autosomal dominant polycystic kidney disease in China. Kidney Dis (Basel) 2016; 2: 111-119.

5. PKD Foundation. Cyst Bleed Saga, https://pkdcure.org/blog/ the-cyst-bleed-saga-2/ (accessed: 17 November 2019).

6. Horie S, Mochizuki T, Muto S, et al. Evidence-based clinical practice guidelines for polycystic kidney disease. Clin Exp Nephrol 2016; 20: 493-509.

7. Lager DJ, Qian Q, Bengal RJ, et al. The PCK rat: a new model that resembles human autosomal dominant polycystic kidney and liver disease. Kidney Int 2001; 59: 126-136.

8. Paka P, Huang B, Dua B, et al. A small molecule fibrokinase inhibitor in a model of fibropolycystic hepatorenal disease. World J Nephrol 2018; 7: 96-107.

9. Zerbinati $\mathrm{N}$ and Calligaro A. Calcium hydroxylapatite treatment of human skin: evidence of collagen turnover through picrosirius red staining and circularly polarized microscopy. Clin Cosmet Investig Dermatol 2018; 11: 29-35.

10. Lee LC, Zhihong Z, Hinson A, et al. Reduction in left ventricular wall stress and improvement in function in failing hearts using Algisyl-LVR. J Vis Exp 2013; doi: 10.3791/50096.

11. Coelho PGB, Souza MV, Conceição LG, et al. Evaluation of dermal collagen stained with picrosirius red and examined under polarized light microscopy. An Bras Dermatol 2018; 93: 415418.

12. Bas O, Nalbant I, Can Sener N, et al. Management of renal cysts. JSLS 2015; 19: e2014.00097.

13. Uemasu J, Fujihara M, Munemura C, et al. Cyst sclerotherapy with minocycline hydrochloride in patients with autosomal dominant polycystic kidney disease. Nephrol Dial Transplant 1996; 11: 843-846. 